\title{
A Direct Correlation between Viscosity and Liquid Structure in Cu-Sn Alloys
}

\author{
Yan Zhao ${ }^{1,2}$ and Xiaoxia Hou ${ }^{1}$ \\ ${ }^{1}$ School of Mechanical and Electronical Engineering, Dezhou University, Dezhou 253023, China \\ ${ }^{2}$ Research Institute of Engineering Materials, Dezhou University, Dezhou 253023, China \\ Correspondence should be addressed to Yan Zhao; dzuzhy@126.com
}

Received 24 August 2017; Accepted 15 November 2017; Published 18 December 2017

Academic Editor: Jan A. Jung

Copyright (c) 2017 Yan Zhao and Xiaoxia Hou. This is an open access article distributed under the Creative Commons Attribution License, which permits unrestricted use, distribution, and reproduction in any medium, provided the original work is properly cited.

\begin{abstract}
The viscosity and liquid structure of $\mathrm{Cu}_{100-x} \mathrm{Sn}_{x}(x=10,20,30,40$, at.\%) melts were investigated. Temperature dependence of viscosity $\eta$ and correlation length $D$ all shows an exponential decay function (Arrhenius-type equation), which is similar to our former studied $\mathrm{Cu}-\mathrm{Ag}$ alloys. The correlation between viscosity and liquid structure had been studied. A simple relation between viscosity and correlation length $D$ was found. The ratio of $D$ and $\eta$ shows a linear relationship with temperature, which is different from our former studied results in $\mathrm{Cu}$-Ag alloys. Among the four $\mathrm{Cu}-\mathrm{Sn}$ alloys, $\mathrm{Cu}_{80} \mathrm{Sn}_{20}$ and $\mathrm{Cu}_{70} \mathrm{Sn}_{30}$ alloys have higher activation energy for viscous flow $\left(E_{a, V}\right)$, activation energy for structural evolution $\left(E_{a, D}\right)$, and the slope $(\gamma)$ of the linear relationship of $D / \eta$ with temperature due to the $\mathrm{Cu}_{3} \mathrm{Sn}$ clusters formation.
\end{abstract}

\section{Introduction}

Viscosity is one of the most important physical properties of melts. It can be seen as the reflection of interaction force in relative motion atoms or molecules, which is also the foundation of the liquid structure. The interatomic interactions change inevitably causes the change of the structure and vice versa. So, viscosity is seen as one of structure sensitive properties [1]. The relationship between viscosity and liquid structure has attracted extensive interests and researches. Earlier in the 1940s, Born and Green [2] set up a correlation between the viscosity of liquid, the pair correlation function $g(r)$, and the pair interatomic potential $\phi(r)$. Successively in the 1950s and 1960s, Zwanzig et al. [3] and Rice and Allnatt [4] put forward a similar equation amended by the friction coefficient $\zeta$ and boundary condition function for finite differential equation $\psi(r)$. Although some results calculated by these models are in good agreement with experiments in some unitary metals, some calculated results are not satisfied due to the difficulty of obtaining the accurate pair interatomic potential $\phi(r)$ [5]. This is especially true for alloys. Few studies on the relationship of viscosity and structure in alloys were reported.
This study is one of our series experimental studies on the correlation between viscosity and liquid structure in binary alloys. The viscosities of $\mathrm{Cu}_{100-x} \mathrm{Sn}_{x}(x=10,20,30,40$, at.\%) melts were investigated by an oscillating viscometer. Based on the structural investigations through X-ray diffraction method [6], a direct correlation between viscosity and liquid structure in $\mathrm{Cu}-\mathrm{Sn}$ alloys was found, which is different from our former studies in Cu-Ag alloys [7].

\section{Material and Methods}

Pure copper (99.99\% mass) and tin (99.98\% mass) were melted in a vacuum induction furnace to prepare the samples of $\mathrm{Cu}-\mathrm{Sn}$ alloys used in this work When the samples cooled to room temperature, they were processed into the suitable size, a cuboid $(25 \mathrm{~mm} \times 18 \mathrm{~mm} \times 8 \mathrm{~mm})$ and a cylinder $(\Phi 28 \mathrm{~mm} \times$ $48 \mathrm{~mm}$ ) for X-ray diffraction and viscosity experiments, respectively.

A high temperature torsional vibration viscometer was used for viscosity investigations. The details of measurement process can be seen in our former works $[8,9]$. An $\theta-\theta$ type X-ray diffractometer of high temperature melts was used for liquid structure experiments. $\mathrm{MoK}_{\alpha}$ radiation 
TABLE 1: Parameters $\eta_{0}, E_{a, V} / R, D_{0}, E_{a, D} / R, \delta$, and $\gamma$ of the Cu-Sn melts.

\begin{tabular}{lccccc}
\hline Alloys & $\eta_{0}(\mathrm{mPa} \cdot \mathrm{s})$ & $E_{a, V} / R(\mathrm{~K})$ & $D_{0}\left(10^{-1} \mathrm{~nm}\right)$ & $E_{a, D} / R(\mathrm{~K})$ & $\gamma\left(10^{-1} \mathrm{~nm} / \mathrm{mPa} \cdot \mathrm{s} \cdot \mathrm{K}\right)$ \\
\hline $\mathrm{Cu}_{90} \mathrm{Sn}_{10}$ & 0.549 & 2033.328 & 5.000 & 624.087 & 0.00253 \\
$\mathrm{Cu}_{80} \mathrm{Sn}_{20}$ & 0.297 & 2604.146 & 3.296 & 1058.307 & 0.00338 \\
$\mathrm{Cu}_{70} \mathrm{Sn}_{30}$ & 0.324 & 2141.963 & 3.328 & 1031.465 & 0.00334 \\
$\mathrm{Cu}_{60} \mathrm{Sn}_{40}$ & 0.637 & 1345.649 & 3.422 & 577.672 & 0.00169 \\
\hline
\end{tabular}

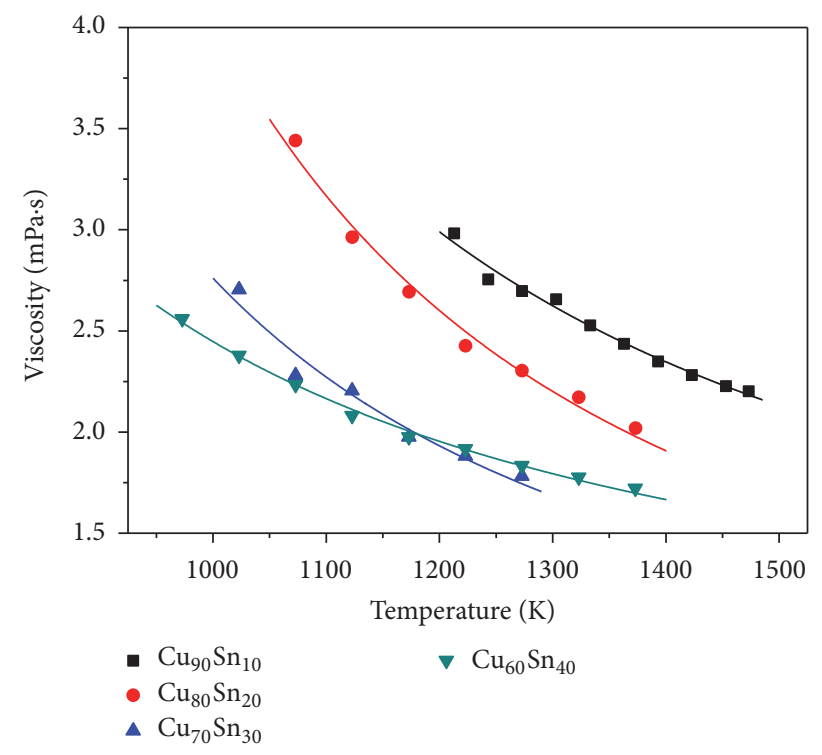

Figure 1: Temperature dependence of viscosities of $\mathrm{Cu}-\mathrm{Sn}$ alloys (the lines are fitted by Arrhenius equation).

(wavelength $\lambda=0.07089 \mathrm{~nm}$ ) diffracted by sample was selected by a graphite monochromator. Firstly, Krogh-MoeNorman method $[10,11]$ was used for the coherent scattering intensity transformation. Secondly, the amended values given by Cromer and Mann [12] were used to correct the Compton scattering. At last, the structure factor $S(Q)$ was obtained through Ashcroft-Langreth method [13, 14]. The detailed process can be seen in $[15,16]$. The correlation length $D$ used in this work was calculated as $D=2 \pi / \Delta Q$, where $\Delta Q$ is the half-height-width of the first peak of $S(Q)[17,18]$.

\section{Results and Discussion}

The measured viscosities (the scattered points) of the four $\mathrm{Cu}-\mathrm{Sn}$ alloys are shown in Figure 1. The changing trend of viscosity with temperature can be fitted well by an Arrhenius equation:

$$
\eta=\eta_{0} \exp \left(\frac{E_{a, V}}{R T}\right),
$$

where $\eta_{0}$ is a constant, which is decided by the nature of the material, $E_{a, V}$ the activation energy for viscous flow, $R$ the gas constant, and $T$ the Kelvin temperature.

From Figure 1, it can be seen that the viscosity of $\mathrm{Cu}-\mathrm{Sn}$ alloys decreases with the increasing of Sn content as a whole.

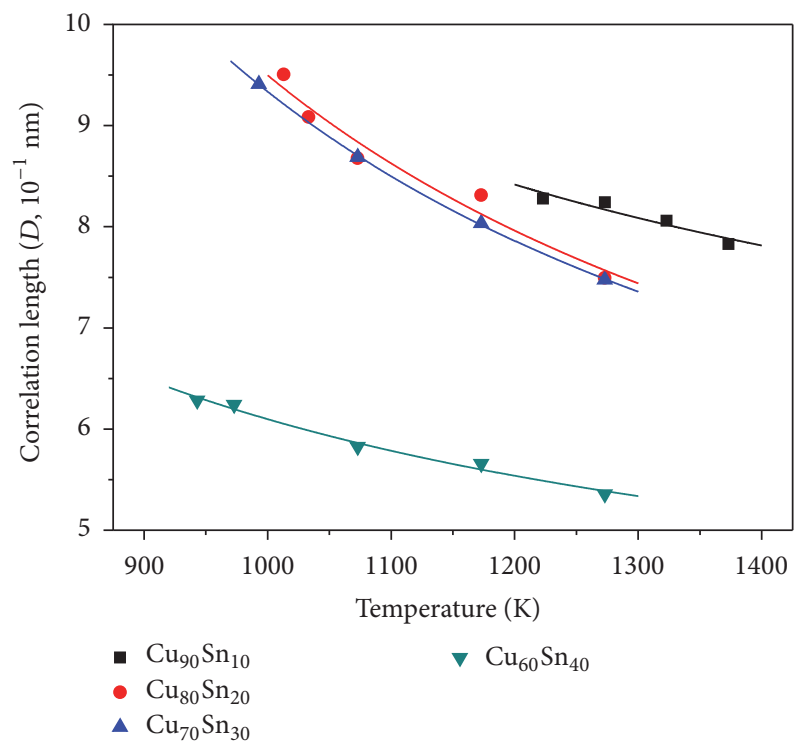

FIGURE 2: Temperature dependence of correlation length of $\mathrm{Cu}-\mathrm{Sn}$ alloys (the lines are fitted by the Arrhenius equation).

But the viscosities of $\mathrm{Cu}_{80} \mathrm{Sn}_{20}$ and $\mathrm{Cu}_{70} \mathrm{Sn}_{30}$ alloys change faster with temperature than the other two alloys. We believe this is associated with the formation of $\mathrm{Cu}_{3} \mathrm{Sn}$ clusters with temperature decreasing in $\mathrm{Cu}-\mathrm{Sn}$ alloys [19]. The values of $\eta_{0}$ and $E_{a, V} / R$ for the four $\mathrm{Cu}-\mathrm{Sn}$ melts can be gotten through fitting the viscosity data in Figure 1 by (1). The detailed fitting results are shown in Table 1 . The results show that $\mathrm{Cu}_{90} \mathrm{Sn}_{10}$ and $\mathrm{Cu}_{60} \mathrm{Sn}_{40}$ alloys have smaller value of $\eta_{0}$ and larger value of $E_{a, V} / R$. And $\mathrm{Cu}_{80} \mathrm{Sn}_{20}$ alloy has the minimum value of $\eta_{0}$ $(0.297 \mathrm{mPa} \cdot \mathrm{s})$ and the maximal value of $E_{a, V} / R(2604.146 \mathrm{~K})$.

The correlation length $D$ (the scattered points) of the studied four $\mathrm{Cu}-\mathrm{Sn}$ alloys is shown in Figure 2. It can be seen that the data of correlation length $D$ also show an exponential function change with temperature, which can be fitted by an Arrhenius-type equation:

$$
D=D_{0} \exp \left(\frac{E_{a, D}}{R T}\right),
$$

where $D_{0}$ is a constant, which is decided by the nature of the material, and $E_{a, D}$ is the activation energy for structural evolution.

Similar to the temperature dependence of viscosity, the correlation length $D$ decreases with increasing of Sn content, and the correlation length $D$ of $\mathrm{Cu}_{80} \mathrm{Sn}_{20}$ and $\mathrm{Cu}_{70} \mathrm{Sn}_{30}$ alloys changes faster with temperature than the other two alloys 
also. Through fitting the correlation length $D$ in Figure 2 by (2), $D_{0}$ and $E_{a, D} / R$ of the four $\mathrm{Cu}$-Sn alloys can be gotten. The detailed fitting results are shown in Table 1 . The results show that $\mathrm{Cu}_{80} \mathrm{Sn}_{20}$ alloy has the minimum value of $D_{0}(0.3296 \mathrm{~nm})$ and the maximal value of $E_{a, D} / R(1058.307 \mathrm{~K})$.

The viscosity $\eta$ and correlation length $D$ for the four studied $\mathrm{Cu}$-Sn alloys all show Arrhenius-type relations with temperature, that is, an exponential decay function with temperature. This is similar to the $\mathrm{Cu}-\mathrm{Ag}$ alloys we studied earlier [7]. Does this mean that the correlations between viscosity $\eta$ and correlation length $D$ in $\mathrm{Cu}-\mathrm{Ag}$ alloys are also fit for $\mathrm{Cu}-\mathrm{Sn}$ alloys? For $\mathrm{Cu}-\mathrm{Ag}$ alloys, the correlations between viscosity $\eta$ and correlation length $D$ were found as [7]

$$
\begin{aligned}
k D-\eta=\alpha \exp \left(\frac{\beta}{R T}\right) & \\
& \alpha=k D_{0}-\eta_{0}, \beta=\frac{E_{a, D}^{2}}{E_{a, V}}, k=1 .
\end{aligned}
$$

By calculation, the correlations between viscosity and correlation length in $\mathrm{Cu}-\mathrm{Ag}$ alloys as shown above are not fit for $\mathrm{Cu}-\mathrm{Sn}$ alloys. As one of the simple eutectic binary alloys, $\mathrm{Cu}-\mathrm{Ag}$ melt is homogeneous; the viscosity and liquid structure with temperature change synchronously. It can be seen in our earlier study that the values of activation energy for viscous flow $\left(E_{a, V}\right)$ and structural evolution $\left(E_{a, D}\right)$ are nearly equal to each other, which means the temperature dependence characteristics of viscosity and correlation length (the exponential changing rules) are similar. But, for the $\mathrm{Cu}$ Sn alloy, its structure is inhomogeneous due to the formation of $\mathrm{Cu}_{3} \mathrm{Sn}$ and $\mathrm{Sn}-\mathrm{Sn}$ clusters [19]. It can be seen from Table 1 that the values of activation energy for viscous flow $\left(E_{a, V}\right)$ and structural evolution $\left(E_{a, D}\right)$ have a greater difference. So the viscosity and liquid structure with temperature change asynchronously in $\mathrm{Cu}-\mathrm{Sn}$ alloy.

In consideration of instability of atomic clusters in high temperature, they have less influence on atomic transport properties, such as the viscosity. But, in low temperature area, atomic clusters are relatively stable, and interaction between them is reinforced, so they have greater influence on viscosity. Namely, relative to the structure change, viscosity changes slowly with temperature in high temperature and changes quickly in low temperature. So the relationship between viscosity and structure should also be related to the temperature. By the study on the ratio of correlation length and viscosity, we found that it is proportional to the temperature, which is shown in Figure 3. The lines are fitted by a linear equation:

$$
\frac{D}{\eta}=\gamma T+c
$$

The slopes $(\gamma)$ of the fitted lines are shown in Table 1 . It can be seen from Figure 3 and Table 1 that the slopes of $\mathrm{Cu}_{80} \mathrm{Sn}_{20}$ and $\mathrm{Cu}_{70} \mathrm{Sn}_{30}$ alloys are larger than the other two $\mathrm{Cu}-\mathrm{Sn}$ alloys. We believe that this is associated with more $\mathrm{Cu}_{3} \mathrm{Sn}$ clusters forming in $\mathrm{Cu}_{80} \mathrm{Sn}_{20}$ and $\mathrm{Cu}_{70} \mathrm{Sn}_{30}$ alloys than the other two.

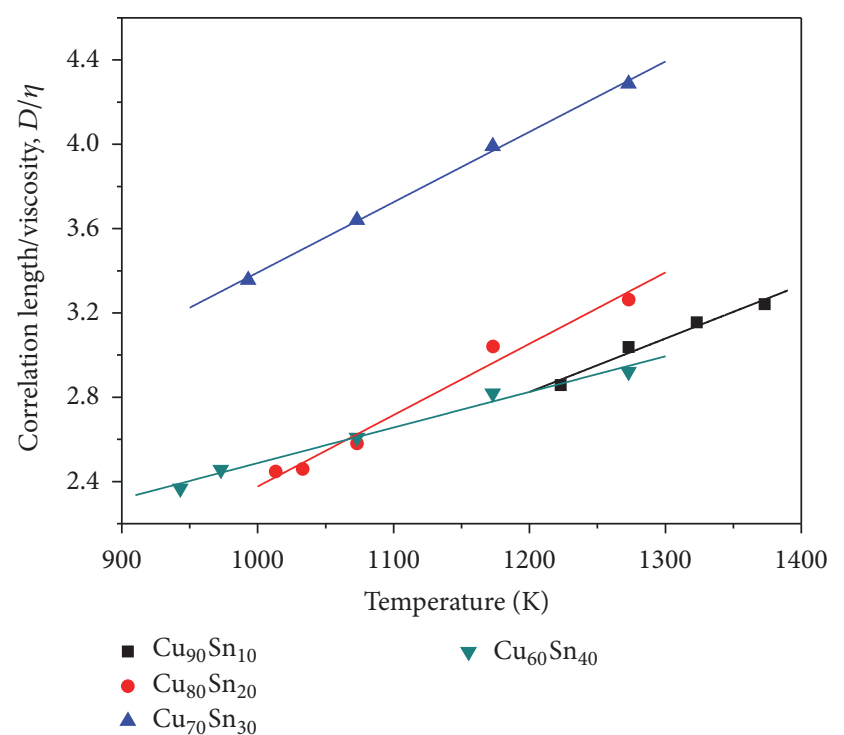

FIGURE 3: Temperature dependence of the ratio of correlation length and viscosity of $\mathrm{Cu}$-Sn alloys (the lines are fitted by a linear equation).

\section{Conclusion}

To summarize, the viscosity and liquid structure of four $\mathrm{Cu}-$ Sn melts were investigated by an oscillating viscometer and $\mathrm{X}$-ray diffraction method. The correlation between viscosity and liquid structure was studied. The results show that temperature dependence of viscosity $\eta$ and correlation length $D$ can be all fitted well by an Arrhenius-type equation. The ratio of correlation length $D$ and viscosity $\eta$ shows a linear relationship with temperature, which is different from our earlier studied results in $\mathrm{Cu}-\mathrm{Ag}$ alloys. Among the four $\mathrm{Cu}-$ Sn alloys, $\mathrm{Cu}_{80} \mathrm{Sn}_{20}$ and $\mathrm{Cu}_{70} \mathrm{Sn}_{30}$ alloys have higher $E_{a, V}$ (the activation energy for viscous flow), $E_{a, D}$ (the activation energy for structural evolution), and $\gamma$ (the slope of the linear relationship of $D / \eta$ with temperature) due to the more $\mathrm{Cu}_{3} \mathrm{Sn}$ clusters formation in $\mathrm{Cu}_{80} \mathrm{Sn}_{20}$ and $\mathrm{Cu}_{70} \mathrm{Sn}_{30}$ melts than the other two $\mathrm{Cu}$-Sn alloys. The results of our work will enhance the understanding of the correlations between dynamic properties and liquid structure of alloy melts.

\section{Conflicts of Interest}

The authors declare that they have no conflicts of interest.

\section{Acknowledgments}

This work was supported by the National Natural Science Foundation of China (Grant no. 51501029) and the Shandong Provincial Natural Science Foundation, China (Grant no. ZR2014EL001).

\section{References}

[1] B. Nikolaev and J. Vollmann, "Viscosity of Ag-Sb alloys," Journal of Non-Crystalline Solids, vol. 208, no. 1-2, pp. 145-150, 1996.

[2] M. Born and H. S. Green, "A general kinetic theory of liquids. III. Dynamical properties," Proceedings of the Royal Society A 
Mathematical, Physical and Engineering Sciences, vol. 190, pp. 455-474, 1947.

[3] R. W. Zwanzig, J. G. Kirkwood, K. F. Stripp, and I. Oppenheim, "The statistical mechanical theory of transport processes. VI. A calculation of the coefficients of shear and bulk viscosity of liquids," The Journal of Chemical Physics, vol. 21, no. 11, pp. 20502055, 1953.

[4] S. A. Rice and A. R. Allnatt, "On the kinetic theory of dense fluids. VI. Singlet distribution function for rigid spheres with an attractive potential," The Journal of Chemical Physics, vol. 34, no. 6, pp. 2144-2155, 1961.

[5] Y. Waseda, The Structure of Non-Crystalline Materials, McGrawGill, New York, NY, USA, 1980.

[6] Y. Zhao, X. Bian, J. Qin, X. Qin, and X. Hou, "Structural evolution in the solidification process of $\mathrm{Cu}-\mathrm{Sn}$ alloys," Journal of Non-Crystalline Solids, vol. 353, no. 52-54, pp. 4845-4848, 2007.

[7] Y. Zhao and X. Hou, "Viscosities and their correlations with structures of $\mathrm{Cu}-\mathrm{Ag}$ melts," Chinese Physics B, vol. 24, no. 9, article 096601, 2015.

[8] Y. Zhao, X. Bian, and X. Hou, "Viscosity and fragility of the supercooled and superheated liquids of the $\mathrm{Ni}_{60} \mathrm{Zr}_{30} \mathrm{Al}_{10}$ metallic glass-forming alloy," Physica A: Statistical Mechanics and its Applications, vol. 367, pp. 42-54, 2006.

[9] P. Si, X. Bian, J. Zhang, H. Li, M. Sun, and Y. Zhao, “The fragility of Al-Ni-based glass-forming melts," Journal of Physics: Condensed Matter, vol. 15, no. 32, pp. 5409-5415, 2003.

[10] J. Krogh-Moe, "A method for converting experimental X-ray intensities to an absolute scale," Acta Crystallographica, vol. 9, no. 11, pp. 951-953, 1956.

[11] N. Norman, "The Fourier transform method for normalizing intensities," Acta Crystallographica, vol. 10, pp. 370-373, 1957.

[12] D. T. Cromer and J. B. Mann, "Compton scattering factors for spherically symmetric free atoms," The Journal of Chemical Physics, vol. 47, p. 1892, 1967.

[13] J. E. Enderby, E. W. Mitchell, and J. G. Powles, "Neutron Diffraction, Isotopic Substitution and the Structure of Aqueous Solutions [and Discussion]," Philosophical Transactions of the Royal Society B: Biological Sciences, vol. 290, no. 1043, pp. 553-566, 1980.

[14] A. C. Barnes, M. A. Hamilton, P. Buchanan, and M.-L. Saboungi, "Combined X-ray and neutron diffraction from binary liquids and amorphous semiconductors," Journal of Non-Crystalline Solids, vol. 250-252, pp. 393-404, 1999.

[15] B. C. Giessen and C. N. J. Wagner, Liquid Metals, Marcel Dekker, New York, NY, USA, 1972.

[16] V. V. Buhalenko, A. G. Ilinskii, and A. V. Romanova, "X-ray diffraction study on the liquid structure of Zn-Ga alloy system," Metallofizika, vol. 13, p. 92, 1991.

[17] A. P. Sokolov, A. Kisliuk, M. Soltwisch, and D. Quitmann, "Medium-range order in glasses: Comparison of Raman and diffraction measurements," Physical Review Letters, vol. 69, no. 10, pp. 1540-1543, 1992.

[18] E. Vateva and E. Savova, "New medium-range order features in Ge-Sb-S glasses," Journal of Non-Crystalline Solids, vol. 192-193, pp. 145-148, 1995.

[19] Y. Zhao, Structural evolution and cluster behavior in the solidification process of binary alloy's melts [Ph. D. Dissertation], Shandong University, 2007. 

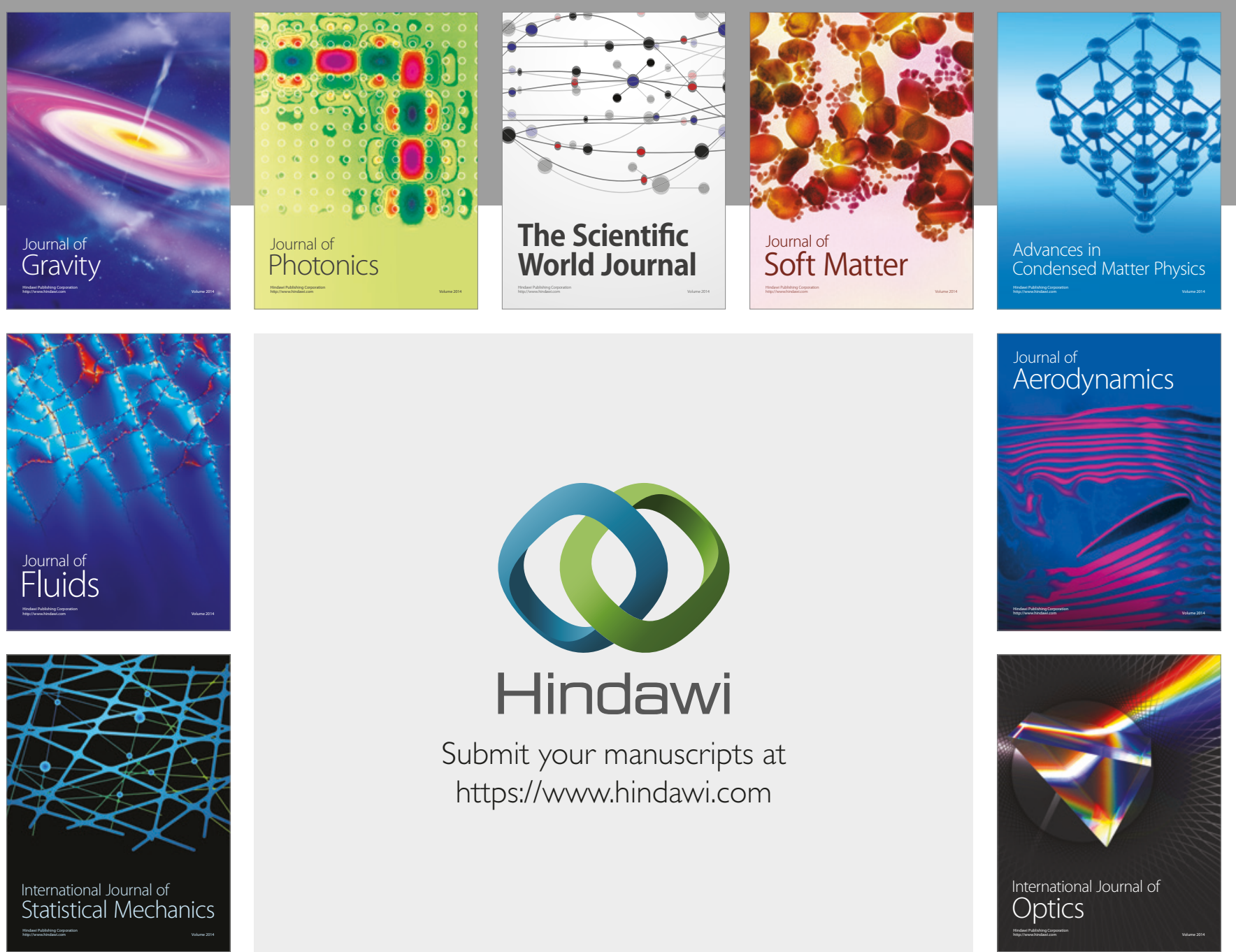

Submit your manuscripts at

https://www.hindawi.com
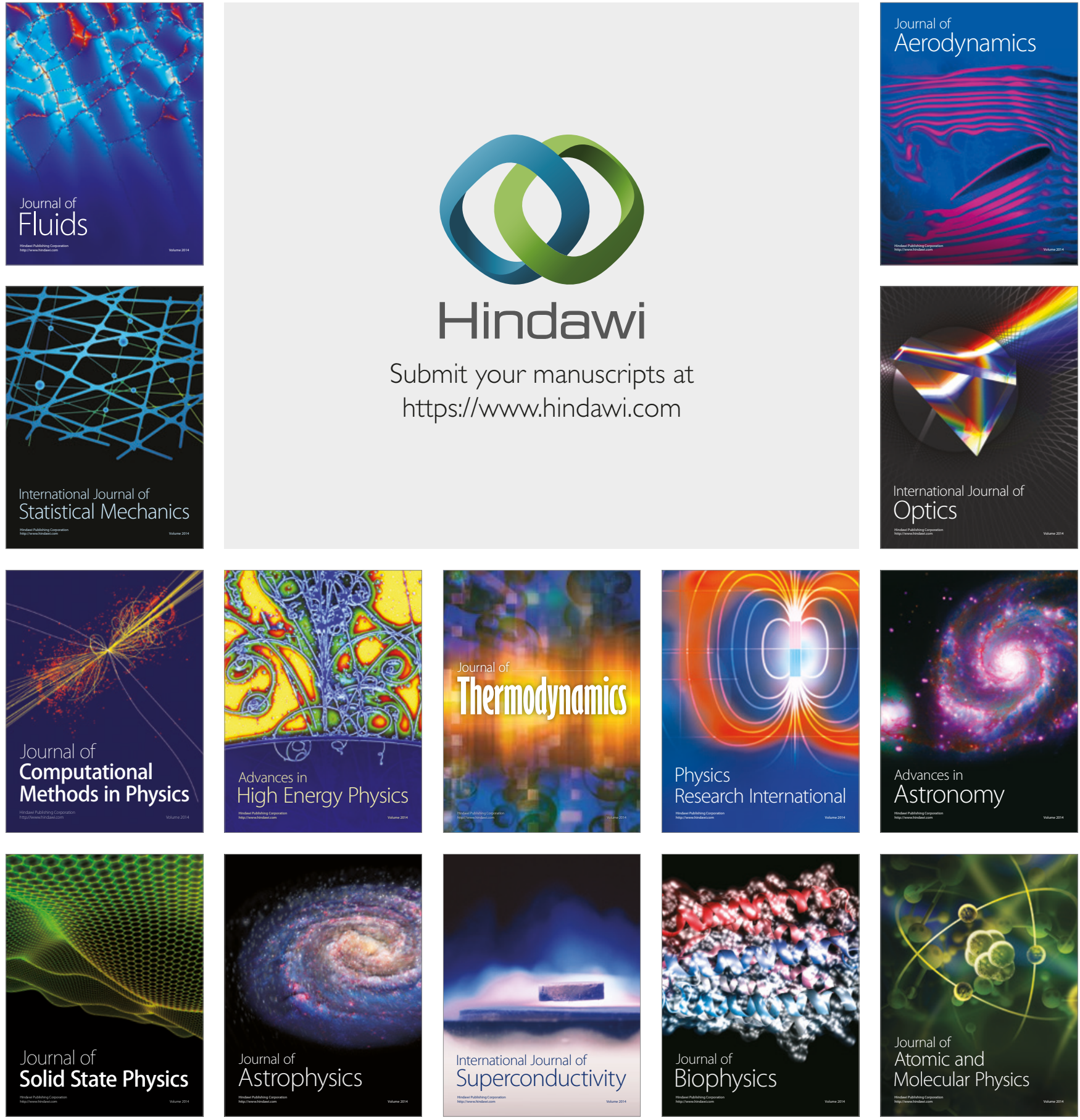